\title{
Falsa broca aumenta disseminação de Beauveria bassiana em populações de campo da broca-do-rizoma da bananeira
}

\author{
West Indian Sugarcane Weevil enhances dissemination of Beauveria bassiana on field populations of \\ the banana weevil
}

\author{
Giuliano Pauli $^{\mathrm{I}, \mathrm{II}}$ Rogério Biaggioni Lopes ${ }^{\mathrm{III}}$ Sérgio Batista Alves ${ }^{\mathrm{I} \dagger}$ Erval Rafael Damatto Junior $^{\mathrm{IV}}$ \\ Gabriel Moura Mascarin
}

\section{- NOTA -}

RESUMO

A transmissão horizontal de fungos entomopatogênicos tem papel importante na epizootiologia, pois está diretamente associada à disseminação do agente causal da doença de insetos. Em hospedeiros que apresentam restrita mobilidade, a presença de pragas secundárias no agroecossistema pode ajudar na formação de focos secundários da doença e, com isso, aumentar a mortalidade das pragaschave. Para tanto, a disseminação de Beauveria bassiana, introduzida inoculativamente através de iscas tipo telha, foi avaliada em um bananal infestado por Cosmopolites sordidus (Piracicaba) e outro por C. sordidus e Metamasius hemipterus (Botucatu). Constatou-se que, em Piracicaba, não foram capturados insetos infectados na testemunha, sendo os índices de infecção nas parcelas que receberam $\boldsymbol{B}$. bassiana de $8 e$ 15\%. Em Botucatu, o índice de infecção dos insetos capturados na testemunha chegou a $17 \%$, enquanto que, nas parcelas tratadas, variou de 28 a 54\%. Os resultados obtidos neste trabalho demostram que a presença de M. hemipterus influenciou na disseminação de B. bassiana para a população de $\boldsymbol{C}$. sordidus, praga-chave dessa cultura.

Palavras-chave: Cosmopolites sordidus, Metamasius hemipterus, transmissão horizontal, epizootiologia.

\section{ABSTRACT}

Horizontal transmission of entomopathogenic fungi plays an important role in the epizootiology, because it is directly associated with the spread of causal agents of insect diseases. In hosts with restricted mobility, the presence of secondary pests in the agroecosystem can help in the formation of secondary disease outbreaks and increase the mortality of the target pest. For this purpose, the dissemination of Beauveria bassiana inoculated using tile-type baits was evaluated in a banana plantation infested with Cosmopolites sordidus (Piracicaba) and one with C. sordidus and Metamasius hemipterus (Botucatu). In Piracicaba, insects contaminated with the pathogen were not found in the control group, and infection rates in the plots that received B. bassiana ranged between 8 and $15 \%$. In Botucatu, the infection rate of insects in the control reached 17\%, whereas in the fungal treatment plots, it varied from 28 to $54 \%$. The present results demonstrate the influence of M. hemipterus in the dissemination of $\boldsymbol{B}$. bassiana in populations of $\boldsymbol{C}$. sordidus, a target pest of this crop.

Key words: Cosmopolites sordidus, Metamasius hemipterus, horizontal transmission, epizootiology.

Cosmopolites sordidus (Germar) (Coleoptera: Curculionidae), a broca-do-rizoma, é a praga mais importante da bananeira, encontrando-se amplamente distribuída por todo o mundo (GOLD et al., 2001). No Brasil, ocorre em todas as regiões produtoras de banana, causando redução no tamanho dos cachos, enfraquecimento e queda de plantas, diminuição na produtividade, além de favorecer o ataque do fungo Fusarium oxysporum, causador do mal-do-Panamá (MESQUITA, 2003; FANCELLI et al., 2004). Apesar de ser uma praga de hábito gregário, com limitada dispersão e raramente alçar voo, mesmo apresentando asas funcionais, esse inseto se dispersa,

'Laboratório de Patologia e Controle Microbiano, Escola Superior de Agricultura "Luiz de Queiroz" (ESALQ), Av Pádua Dias 11, 13418-900, Piracicaba, SP, Brasil. E-mail: giulianopauli@yahoo.com.br. *Autor para correspondência. ${ }^{\dagger}$ In memorian.

II FMC Química do Brasil, Campinas, SP, Brasil.

III EMBRAPA Cenargen, Brasília, DF, Brasil.

rv Agência Paulista de Tecnologia dos Agronegócios, Registro, SP, Brasil.

vEMBRAPA Arroz e Feijão, Santo Antônio de Goiás, GO, Brasil. 
principalmente, através de materiais infestados com ovos, larvas, pupas e/ou adultos, ocorrendo a dispersão de forma passiva (GOLD et al., 1998).

Metamasius hemipterus (Olivier) (Coleoptera: Curculionidae), a falsa-broca-dabananeira, é referida por não causar dano econômico à cultura da banana, apesar de se alimentar dessa planta, geralmente, em pseudocaules tombados, em decomposição ou em plantas debilitadas. Entretanto, quando em alta população, pode causar prejuízos à bananeira, como observado em algumas regiões (PEREIRA et al., 2004). M. hemipterus, diferente de $\boldsymbol{C}$. sordidus, apresenta grande mobilidade na lavoura, utilizando-se do voo como meio de locomoção e dispersão. Esse comportamento faz de M. hemipterus um inseto eficiente como agente de disseminação de entomopatógenos, levando inóculo de fungos para cultivos vizinhos com populações de insetos isentas da doença (DOLINSKI \& LACEY, 2007).

No Brasil, desde o final da década de 1980, vêm sendo estudadas formas de disseminação de fungos entomopatogênicos para o controle de $\boldsymbol{C}$. sordidus, principalmente por meio de iscas atrativas (MESQUITA, 1988; BATISTA FILHO et al., 1991; FANCELLI et al., 2004). Destaque tem sido dado para o fungo entomopatogênico Beauveria bassiana (Bals.) Vuill. (Ascomycota: Cordycipitaceae), conhecido por causar a doença muscardine branca em diversos insetos. Diversos estudos realizados em laboratório têm evidenciado a suscetibilidade da broca-do-rizoma a determinados isolados desse fungo (BATISTA FILHO et al., 1991; JORDÃO et al., 1999; GOLD et al., 2001; FANCELLI et al., 2004; ALMEIDAet al., 2009).

O fungo $\boldsymbol{B}$. bassiana não causa epizootias em áreas de cultivo de banana e, mesmo ocorrendo frequentemente sobre as populações de insetos, esse fungo apresenta baixos índices de infecção natural (GOLD et al., 2001). Dessa forma, aplicações desse patógeno são necessárias para que haja o estabelecimento da doença no campo, proporcionando, em longo prazo, o controle dessa praga. O uso de iscas atrativas de pseudocaule é prática frequente, sendo realizada com sucesso em países da América Latina e África (GODONOU et al., 2000; FANCELLI et al., 2004). Apesar disso, o uso de iscas vegetais atrativas, associadas a conídios de B. bassiana, apresenta algumas limitações e resultados controversos (GOLD et al., 2001).

Muitos fatores podem afetar a dinâmica da doença, causada por B. bassiana introduzido inoculativamente em condições de campo, sobre a população da broca. Fatores abióticos, como temperatura e umidade da região e suas variações sazonais, influenciam diretamente na ocorrência da doença, além de aspectos relacionados à aplicação do fungo, como a frequência, a quantidade de propágulos e o método de aplicação. Também é importante a presença de hospedeiros secundários, alternativos e intermediários que, além de favorecerem a variabilidade do patógeno, são fundamentais para a manutenção do inóculo no ambiente, principalmente em agroecossistemas (monocultivos) (ALVES \& LECUONA, 1998).

O objetivo deste trabalho foi avaliar a ocorrência de transmissão horizontal do fungo $\boldsymbol{B}$. bassiana entre adultos da falsa-broca-da-bananeira, $\boldsymbol{M}$. hemipterus, e da broca-do-rizoma $C$. sordidus. Para tanto, foram selecionadas duas áreas experimentais, uma contendo apenas $\boldsymbol{C}$. sordidus e a outra contendo simultaneamente a presença de $\boldsymbol{C}$. sordidus e de $\boldsymbol{M}$. hemipterus. As áreas experimentais eram cultivadas com banana 'Nanica' e apresentavam cerca de cinco anos de idade. Uma das áreas experimentais foi instalada na região de Botucatu e a outra em Piracicaba, Estado de São Paulo. Em ambas as áreas, foram demarcadas quatro faixas de, aproximadamente, $100 \mathrm{~m}^{2}$ cada, correspondentes às parcelas nas quais os experimentos foram instalados e onde foram introduzidos ou não conídios de $\boldsymbol{B}$. bassiana (Boveril ${ }^{\circledR}$ WP, isolado ESALQ-PL63).

Para a avaliação da população de insetos, foram instaladas, nas parcelas das duas áreas experimentais, seis iscas do "tipo telha" constituídas por pseudocaule de bananeira (MESQUITA, 2003), visando a determinar o índice de infestação das duas espécies de insetos e a incidência natural de fungos entomopatogênicos sobre os adultos. Após essa avaliação preliminar, foram instaladas, nas mesmas áreas, iscas "tipo telha", contendo ou não B. bassiana . As iscas foram instaladas no período entre março e abril de 2007, sendo realizadas duas aplicações com intervalos de, aproximadamente, 21 dias, instalando-se novas iscas tratadas no mesmo local. Para ambas as áreas, a inoculação das iscas foi feita com $15 \mathrm{~g}$ do produto microbiano formulado com pó de amido de arroz na concentração de $1 \times 10^{9}$ conídios viáveis $\mathrm{g}^{-1}$, aplicado sobre a face cortada da isca.

As iscas foram distribuídas de forma equidistante nas parcelas das áreas experimentais, sendo colocadas na proporção de 600 por hectare (seis iscas por parcela). Foram posicionadas próximas à base das plantas, com a face tratada voltada para o solo, onde permaneceram cobertas com folhas de bananeira pelo período de 21 dias. Após esse período, as iscas foram recolhidas e os insetos vivos retirados, colocados em recipientes plásticos com tampa e alimentados com pedaços de pseudocaule pelo período 
de três semanas. Os insetos mortos foram colocados diretamente em câmara úmida para confirmação da mortalidade causada pelo patógeno.

Os experimentos foram desenvolvidos em delineamento inteiramente aleatorizado e em faixas. Os dados foram submetidos à análise não-paramétrica de Kruskal-Wallis (PROC NPAR1WAY) e as médias comparadas através do teste de Bonferroni-Dunn (PROC GLM) a 5\% de probabilidade (SAS INSTITUTE INC, 2004).

Em Piracicaba, foi capturado somente $\boldsymbol{C}$. sordidus, em número total de 403, enquanto que em Botucatu foram coletados $334 \boldsymbol{C}$. sordidus $(46,8 \%)$ e 389 M. hemipterus $(53,8 \%)$ (Tabela 1$)$. As condições climáticas observadas nas duas cidades avaliadas, nos meses em que foram realizados os experimentos, não apresentaram diferenças marcantes que pudessem justificar variações na dinâmica da doença no campo. Em Piracicaba, ocorreu temperatura mínima de $17,8^{\circ} \mathrm{C}$, máxima de $31,7^{\circ} \mathrm{C}$, média de $23,8^{\circ} \mathrm{C}$ e precipitação média de $61,2 \mathrm{~mm}$ e, em Botucatu, verificou-se temperatura mínima de $18^{\circ} \mathrm{C}$, máxima de $30,2^{\circ} \mathrm{C}$, média $23,9^{\circ} \mathrm{C}$ e precipitação média de $65,8 \mathrm{~mm}$.

$\mathrm{Na}$ avaliação preliminar realizada em Botucatu e em Piracicaba, o número médio de insetos capturados (não-infectados) por isca, em ambas as áreas, foi de 4,4, demonstrando que a densidade inicial de insetos não foi diferente para as duas áreas (Botucatu: $\mathrm{F}_{\text {prévia }}=0,24 ; P=0,6275$; Piracicaba: $\mathrm{F}_{\text {prevvia }}=1,43$; $P=0,2489)$. Nessa mesma avaliação, em ambas as áreas experimentais, não foram capturados insetos infectados com fungos entomopatogênicos nativos, indicando a não ocorrência natural do patógeno nessas áreas. Não foi observada redução no número de insetos capturados durante o período experimental para as duas áreas (Piracicaba: $\mathrm{F}_{1^{a} \text { avalią̧ão }}=0,88 ; \quad P=0,3616$;
$\mathrm{F}_{2^{\mathrm{a}} \text { avaliação }}=1,96 ; P=0,1803 ;$ Botucatu: $\mathrm{F}_{1^{\mathrm{a}} \text { avaliação }}=0,46$; $\left.P=0,5031 ; \mathrm{F}_{2 \text { avaliąăa }}=0,75 ; P=0,3925\right)$, tanto nas parcelas tratadas como naquelas não tratadas com o fungo, o que indica que esse entomopatógeno não reduziu significativamente a população da praga nas áreas onde foi aplicado.

Após a colocação de iscas tratadas com o fungo, insetos doentes foram capturados nas parcelas tratadas em Piracicaba, com índices de infecção de 15\% para a primeira avaliaçãoe de $8 \%$ para a segunda (Tabela 1). Em Botucatu, os índices foram de $28 \%$ na primeira e de $54 \%$ na segunda avaliação, sendo que nesta última foi constatada diferença significativa entre os locais $\left(\mathrm{F}_{2^{\text {avvaliação }}}=29,39 ; P<0,0001\right)$. Em Botucatu, $17 \%$ dos insetos da testemunha apareceram infectados com o patógeno na segunda avaliação, enquanto que em Piracicaba este fato não foi constatado $\left(\mathrm{F}_{2^{\mathrm{a}} \text { avaliação }}=5,02\right.$; $P=0,0366$ ). Em Piracicaba, foi verificado um aumento no número de insetos capturados nas iscas dispostas nas parcelas da testemunha, o que não foi constatado em Botucatu, ocorrendo diferença significativa entre as áreas na segunda avaliação $\left(\mathrm{F}_{2^{2} \text { avalią̧ão }}=21,50\right.$; $P=0,0002)$. Do total de insetos coletados em Botucatu (334 C. sordidus e 389 M. hemipterus), aproximadamente $76 \%$ dos infectados pertenciam a $\boldsymbol{M}$. hemipterus e apenas $24,1 \%$ a $\boldsymbol{C}$. sordidus, mesmo sendo semelhantes as populações das duas espécies de brocas na área (Tabela 1).

A diferença no número de insetos infectados com B. bassiana em Botucatu, em relação a Piracicaba, pode ser explicada pela presença de M. hemipterus, que ocorreu apenas em Botucatu, e também pela maior atividade ou mobilidade dessa espécie de inseto, já que, inicialmente, o número de insetos das duas espécies em ambas as áreas foi semelhante (Tabela 1). A presença de insetos infectados com o fungo nas

Tabela 1 - Número médio de insetos de Cosmopolites sordidus e Metamasius hemipterus capturados por armadilhas em áreas de produção de banana localizadas em Botucatu e Piracicaba, e incidência de insetos doentes após introdução inoculativa de Beauveria bassiana.

\begin{tabular}{|c|c|c|c|c|c|c|c|}
\hline \multirow{2}{*}{ Área experimental } & \multirow{2}{*}{ Tratamento } & \multicolumn{3}{|c|}{------Insetos capturados por armadilha ${ }^{1}------$} & \multicolumn{3}{|c|}{-------------Insetos doentes $(\%)^{1}------------$} \\
\hline & & Prévia & $1^{\underline{\mathrm{a}}}$ aval. & $2^{\mathrm{a}}$ aval. & Prévia & $1^{\mathrm{a}}$ aval. & $2^{\mathrm{a}}$ aval. \\
\hline \multirow[b]{2}{*}{ Botucatu } & Testemunha & $4,6 \pm 0,9 \mathrm{~A} \mathrm{a}$ & $5,0 \pm 1,1 \mathrm{~A} \mathrm{a}$ & $5,6 \pm 0,9 \mathrm{~A} \mathrm{a}$ & $0,0 \pm 0,0 \mathrm{~A} \mathrm{a}$ & $9,9 \pm 3,8 \mathrm{~A} \mathrm{a}$ & $17,3 \pm 7,1 \mathrm{~B} \mathrm{a}$ \\
\hline & $\begin{array}{l}\text { Beauveria } \\
\text { bassiana }\end{array}$ & $4,3 \pm 1,3 \mathrm{~A} \mathrm{a}$ & $6,8 \pm 1,8 \mathrm{~A} \mathrm{a}$ & $8,5 \pm 1,6 \mathrm{~A} \mathrm{a}$ & $0,0 \pm 0,0 \mathrm{~A} \mathrm{a}$ & $28,2 \pm 6,6 \mathrm{~A} \mathrm{a}$ & $54,2 \pm 5,9 \mathrm{~B} \mathrm{~b}$ \\
\hline \multirow[b]{2}{*}{ Piracicaba } & Testemunha & $5,2 \pm 1,1 \mathrm{~A} \mathrm{a}$ & $8,2 \pm 1,8 \mathrm{~A} \mathrm{a}$ & $14,5 \pm 2,3 \mathrm{~B} \mathrm{a}$ & $0,0 \pm 0,0 \mathrm{~A} \mathrm{a}$ & $0,0 \pm 0,0 \mathrm{~A}$ a & $0,0 \pm 0,0 \mathrm{~A} \mathrm{a}$ \\
\hline & $\begin{array}{l}\text { Beauveria } \\
\text { bassiana }\end{array}$ & $3,6 \pm 0,6 \mathrm{~A} \mathrm{a}$ & $6,6 \pm 0,9 \mathrm{~A}$ a & $9,5 \pm 1,7 \mathrm{~A}$ a & $0,0 \pm 0,0 \mathrm{~A} \mathrm{a}$ & $15,1 \pm 8,3 \mathrm{~A} \mathrm{a}$ & $8,7 \pm 4,5 \mathrm{~A} \mathrm{a}$ \\
\hline
\end{tabular}

${ }^{1}$ Médias ( $\pm \mathrm{EP}$, erro padrão) seguidas por letras maiúsculas (comparação entre locais dentro do mesmo tratamento e no mesmo período de avaliação) e letras minúsculas (comparação entre tratamentos dentro do mesmo local e no mesmo período de avaliação) distintas são significativamente diferentes pelo teste de Bonferroni-Dunn $(P=0,05)$. O fungo Beauveria bassiana foi aplicado em isca do tipo "telha" de bananeira, empregando 15g de Boveril WP (isolado ESALQ-PL63) por isca, na concentração de $1 \times 10^{9}$ conídios viáveis $\mathrm{g}^{-1}$ de produto. 
parcelas das testemunhas de Botucatu é um indicativo de que a disseminação desse patógeno entre as parcelas ocorreu devido à movimentação de $\boldsymbol{M}$. hemipterus. A transmissão da doença entre indivíduos de $\boldsymbol{C}$. sordidus já foi observada (GODONOU et al., 2000; TINZAARA et al., 2007), apesar de essa espécie apresentar baixa mobilidade. Entretanto, neste trabalho, não foi constatada a disseminação da doença da área tratada para a testemunha em Piracicaba, onde a população de insetos estava composta exclusivamente por $\boldsymbol{C}$. sordidus.

Com base nesses resultados, é possível concluir que a presença de $M$. hemipterus em bananais infestados por C. sordidus pode promover a disseminação de conídios de $\boldsymbol{B}$. bassiana. Isso quando esses forem aplicados de forma inoculativa por meio de iscas atrativas, aumentando o número de focos secundários da doença, o que, a longo prazo, pode aumentar a eficiência dessa estratégia de controle.

\section{REFERÊNCIAS}

ALMEIDA, A.M.B. et al. Seleção de isolados de Beauveria bassiana para controle de Cosmopolites sordidus (Germar, 1824) (Coleoptera: Curculionidae). Arquivos do Instituto Biológico, v.76, n.3, p.489-493, 2009.

ALVES, S.B.; LECUONA, R.E. Epizootiologia aplicada ao controle microbiano de insetos. In: ALVES, S.B. (Ed.). Controle microbiano de insetos. Piracicaba: FEALQ, 1998. Cap.2, p.97-169.

BATISTA FILHO, A. et al. Utilização de Beauveria bassiana (Bals.) Vuill. no controle do moleque da bananeira Cosmopolites sordidus Germar, 1824. Revista Brasileira de Fruticultura, v.13, p.35-40, 1991.

DOLINSKI, C.; LACEY, L.A. Microbial control of arthropod pests of tropical tree fruits. Neotropical Entomology. v.36, n.2, p.161-179, 2007. Disponível em: <http://www.scielo.br/ pdf/ne/v36n2/a01v36n2.pdf>. Acesso em: 20 dez. 2009. doi: 10.1590/S1519-566X2007000200001.

FANCELLI, M. et al. Controle biológico de Cosmopolites sordidus (Germ.) (Coleoptera: Curculionidae) pelo fungo Beauveria bassiana (Bals.) Vuill. Cruz das Almas. Embrapa Mandioca e Fruticultura. 2004. 3p. (Comunicado Técnico, 102).
GODONOU, I. et al. Field evaluation of selected formulations of Beauveria bassiana for the management of the banana weevil (Cosmopolites sordidus) on plantain (Musa sp., AAB group). Biocontrol Science and Technology, v.10, p.779788, 2000. Disponivel em: <http://www.tandfonline.com/doi/ abs/10.1080/09583150020011726>. Acesso em: 15 jan. 2010. doi: $10.1080 / 09583150020011726$.

GOLD, C.S. et al. Recent advances in banana weevil biology, population dynamics and pest status with emphasis on East Africa. In: FRISON, E.A. et al. (Ed.). Mobilizing IPM for sustainable banana production in Africa. Neuspruit: Inibap, 1998. 356p.

GOLD, C.S. et al. Biology and integrated pest management for the banana weevil Cosmopolites sordidus (Germar) (Coleoptera: Curculionidae). Integrated Pest Management Reviews, v.6, p.79-155, 2001. Disponivel em: <http://www.springerlink.com/ content/w147k124413263w8/>. Acesso em: 15 nov. 2009 doi: 10.1023/A: 1023330900707 .

JORDÃO, A.L. et al. Caracterização e eficiência de isolados de Beauveria bassiana (Bals.) Vuill. no controle de Cosmopolites sordidus. Arquivos do Instituto Biológico, v.66, n.2, p.107111, 1999.

MESQUITA, A.L.M. Controle biológico das brocas da bananeira Cosmopolites sordidus (Germar, 1824) e Metamasius hemipterus (Linne, 1764) com fungos entomógenos. In: REUNION DE LA ACORBAT, 8., 1987, Santa Marta. Memórias... Santa Marta: AUGURA, 1988. p.311-324.

MESQUITA, A.L.M. Importância e métodos de controle do "Moleque" ou Broca-do-Rizoma-da-Bananeira. Fortaleza: Embrapa Agroindústria Tropical, 2003. 6p. (Circular Técnica 17).

PEREIRA, P.R.V.S. et al. Ocorrência de Metamasius hemipterus (Linnaeus, 1758) (Coleoptera: Curculionidae) em bananais do estado de Roraima. Boa Vista: Embrapa Roraima. 2004. 6p. (Comunicado Técnico 13).

SAS INSTITUTE INC. SAS user's guide: SAS/STAT ${ }^{\circledR}$, release 9.1. Cary, North Carolina, 2004. 824p.

TINZAARA, W. et al. The use of aggregation pheromone to enhance dissemination of Beauveria bassiana for the control of the banana weevil in Uganda. Biocontrol Science and Technology, v.17, n.2, p.111-124, 2007. Disponivel em: <http://www.tandfonline.com/doi/abs/10.1080/ 09583150600937089\#preview>. Acesso em: 12 nov. 2009. doi: $10.1080 / 09583150600937089$. 